TP Periodica Polytechnica

Transportation Engineering

43(1), pp. 35-47, 2015

DOI: 10.3311/PPtr.7467

Creative Commons Attribution (i)

RESEARCH ARTICLE

\section{Systematic Model Simplification Procedure Applied to an Electro- Pneumatic Clutch Model}

\author{
Barna SZIMANDL ${ }^{1, *}$, Huba NÉMETH ${ }^{1}$
}

ReCEIVEd 04 ApRIL 2014; ACCEPTED AfTER REVISION 30 MAY 2014

\begin{abstract}
In this paper a systematic approach is presented for model simplification. The approach is applied for simplifying a detailed dynamic hybrid model of an electro-pneumatic clutch system that was originally developed from first engineering principles. The model simplification is performed by applying simplifying assumptions to the model. The top-down way of traversing the model elements using their hierarchy tree ensures the systematic way of the simplification. With this systematic approach the resulted simplified models preserve the physical meaning of the variables and parameters, while their complexity is decreased significantly.
\end{abstract}

\section{Keywords}

electro-pneumatic clutch, dynamic hybrid model, systematic model simplification, hierarchical structure of models, variable structure graph of models

\footnotetext{
${ }^{1}$ Department of Automobiles and Vehicle Manufacturing, Faculty of Transportation Engineering and Vehicle Engineering, Budapest University of Technology and Economics, H-1521 Budapest, Hungary

*Corresponding author, e-mail: szimandl.barna@auto.bme.hu
}

\section{Introduction}

Dynamic system models constructed for dynamic simulation purposes, where one of the modeling goal is the high accuracy, are usually too detailed and complex for other applications e.g. for model based control design. Moreover the integration of this kind of models into other ones further increases the complexity, which increases the computational time and the memory claim of the simulation as well. Therefore, the model, which is systematically derived using e.g., the seven step model building procedure, reported in (Hangos, Cameron, 2001), should often be simplified by reducing its size and complexity.

There is a well-known model reduction technique for linear time-invariant state-space models that is based on controllability and observability indices, called grammians and on linear state transformations to construct a balanced realization (Laub et al., 1987; Moore, 1981). Furthermore, this method is generalized for stable nonlinear systems (Hahn, Edgar, 2002; Hahn, Edgar et al. 2003). Unfortunately, these methods are purely black-box in nature because the physical meaning of the state variables and parameters in the reduced model is completely lost. As opposed to the above methods the model structure simplification techniques, reported in (Lakner et al. 1999; Leitold et al. 2002) offer a grey-box alternative to model reduction for lumped dynamic models using the hierarchical structure of the model elements. A top-down way of traversing the model elements using their hierarchy tree offers a systematic way of doing model reduction, which can be done more efficiently instead of using heuristics (Lakner et al., 2005). The above method is applied in (Németh et al., 2005) to obtain a simplified model of a single protection valve in an electronic brake system, where the model elements to be left out are selected based on simulation experiment and engineering insight.

In this paper this systematic model simplification procedure is applied to a dynamic hybrid model of an electro-pneumatic clutch system to derive simplified models for simulation in which the computational time and the memory claim are decreased and as well as for control design purpose. 


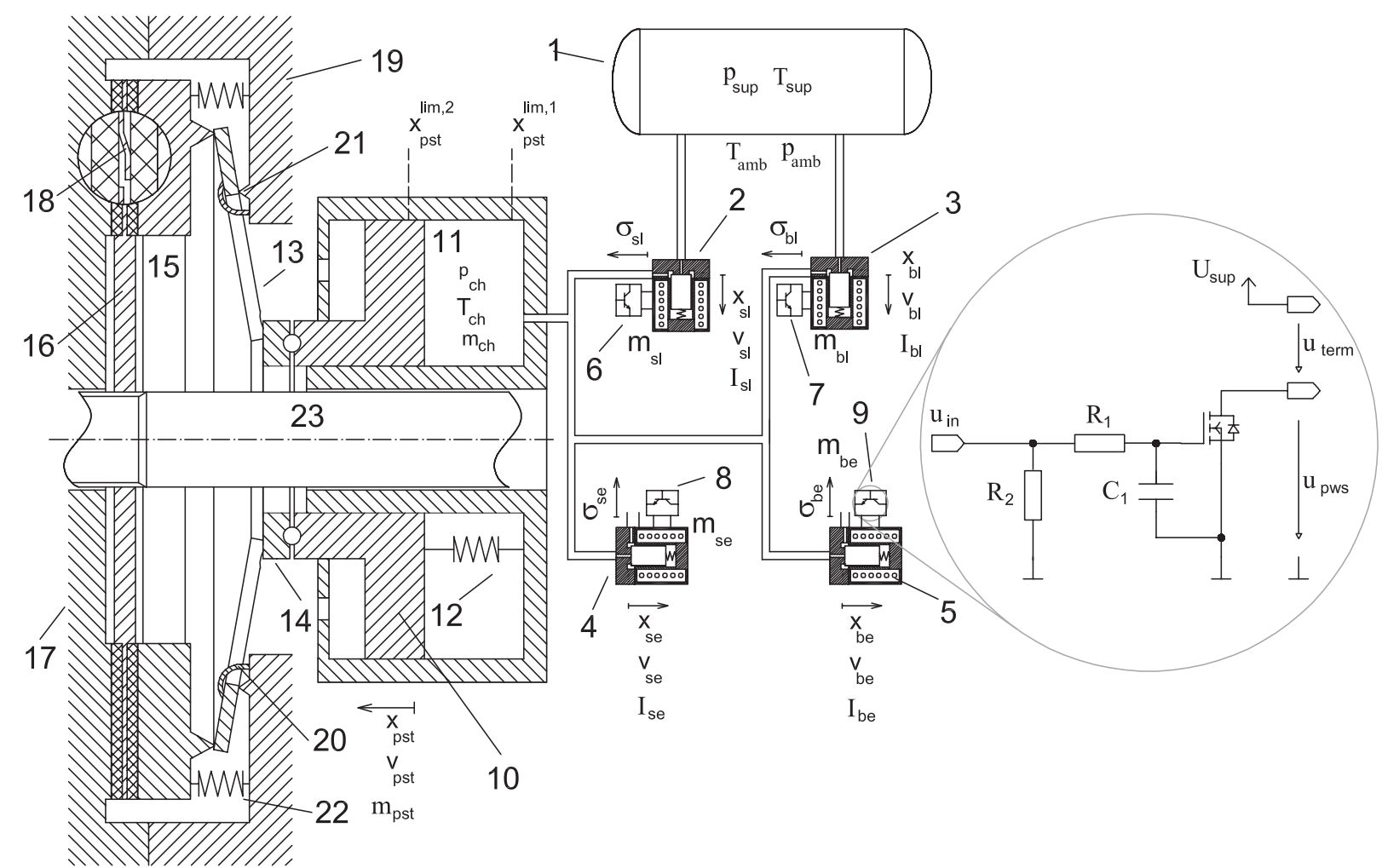

Fig. 1 The layout of the electro-pneumatic clutch (EPC) system

\section{System definition}

In a vehicle driveline, when gear change is demanded, the connection between the engine and the gearbox must be disengaged before any gear shifting procedure is started. This process along with the reconnection of the engine and the gearbox is done by the clutch. The connection is disengaged in the connection point of the engine shaft and the gearbox input shaft, where normally the clutch transmits the momentum through the clutch disc. The clutch friction disc, the pressure plate and the flywheel are rotating together due to the friction force between them. This force is caused by the normal force of a disc spring, which pushes the clutch pressure plate to the friction disc and the flywheel. When the clutching is demanded, Solenoid Magnet Valves (abbreviated as SMV throughout the paper) driven electro-pneumatic actuator pre-stress the disc spring, which lets the clutch pressure plate to move apart from the friction disc, thus terminates the connection. The general layout of the electro-pneumatic clutch (EPC) system (Förster et al. 2004; Förster, Steinel, 2007) with its close surrounding can be seen in Fig. 1.

The system is supplied by compressed air, thus for supply pressure an air reservoir (1) is applied. The actuator contains four SMVs, two of them $(2,3)$ can connect the supply pressure to the chamber (11), so they are called load valves and the remaining two $(4,5)$ can connect the chamber to the ambient pressure, so they are called exhaust valves. The geometry of the four SMVs is identical except for their cross section. The variables of the SMVs are denoted as follows: $s l, b l$, se and be for small- and big load and small- and big exhaust respectively. Each SMV has an own power stage (6-9), which can transform the command signal to appropriate terminal voltage for the solenoid of the SMV.

This structure ensures positive and negative direction displacement of the piston (10), which is the final element of the actuator that performs the clutching procedure. The variables of the piston are denoted by $p s t$ subscript. The actuator contains a holder spring (12), which pushes the piston to the clutch mechanism to reduce the clearance between them. The main load of the actuator comes from the disc spring (13) of the clutch mechanism and acts against the piston movement. The disc spring is slotted in the inner diameter and the release bearing of the piston (14) is connected to this area. The slots have the effect of reducing the spring load and increasing the deflection. In the outer diameter of the disc spring is connected to the pressure plate (15), which can push the clutch friction disc (16) to the flywheel (17). Moreover, the clutch friction disc contains cushion springs (18). The non-linear stiffness of this set of springs has a paramount role in the controllability performance at small torques. The disc spring is compressed between the pressure plate and the housing (19). The disc spring is fixed to the housing with pins (20), which ensures a fulcrum ring (21) where the spring can bend. The pressure plate is also fixed to the housing with tangential leaf springs (22), these springs transform the torque to the housing, determine the radial position of the pressure plate and push it to the disc spring. Finally the flywheel is connected to the engine and the friction disc is connected to the splined gearbox input shaft (23). 
2.1 Detailed nonlinear dynamic hybrid model $\left(\mathcal{M}_{0}\right)$

Since the state space realization is not unique, many equivalent representations with the same dimension can be found, giving rise to the same input-output description of a given system. Hence for the EPC model the following system variables are composed to retain the physical meaning of the variables (see details in Szimandl, Németh, 2013). From the DAEs of the model the state vector is composed of the differential variables as follows:

$$
\begin{gathered}
\mathbf{x}_{\mathcal{M}_{0}}=\left[i_{s l} v_{s l} x_{s l} i_{b l} v_{b l} x_{b l} i_{s e} v_{s e} x_{s e}\right. \\
\left.i_{b e} v_{b e} x_{b e} m_{c h} p_{c h} v_{p s t} x_{p s t}\right]^{T}
\end{gathered}
$$

where $i_{x x}, v_{x x}$ and $x_{x x}$ denote the solenoid current, the armature velocity and the stroke of the corresponding SMV respectively. Moreover, $m_{c h}$ is the air mass in the chamber, $p_{c h}$ is the pressure of the air, $v_{p s t}$ and $x_{p s t}$ are the velocity and the stroke of the piston. The control input vector includes the duty cycle of the PWM control signals of the SMVs:

$$
\mathbf{u}_{\mathcal{M}_{0}}=\left[\begin{array}{llll}
m_{d, s l} & m_{d, b l} & m_{d, s e} & m_{d, b e}
\end{array}\right]^{T}
$$

The uncontrollable inputs form the disturbance vector including the supply voltage, compressed (supply) air- pressure and temperature, ambient- pressure and temperature respectively:

$$
\mathbf{d}_{\mathcal{M}_{0}}=\left[\begin{array}{lllll}
U_{\text {sup }} & p_{\text {sup }} & T_{\text {sup }} & p_{\text {amb }} & T_{a m b}
\end{array}\right]^{T}
$$

The following hybrid nonlinear state space form (Branicky, 1998) of the DAEs of the model is considered:

$$
\frac{d \mathbf{x}_{\mathcal{M}_{0}}^{(k)}}{d t}=\mathbf{f}_{\mathcal{M}_{0}}^{(k)}\left(\mathbf{x}_{\mathcal{M}_{0}}^{(k)}, \mathbf{u}_{\mathcal{M}_{0}}^{(k)}, \mathbf{d}_{\mathcal{M}_{0}}^{(k)}\right)
$$

where $k: \mathbb{R}^{n} \rightarrow \mathbb{N}$ is a piece-wise constant switching function mapping from the state space to $\mathbb{N}$. The nonlinear state functions are written as (the entries that depend on the hybrid modes are boxed and the meaning of the symbols can be found in Table 1 and Table 2):

$$
\begin{aligned}
f_{1, \mathcal{M}_{0}}^{(k)}= & -\frac{v_{s l} i_{s l}}{\left(\mathcal{R}_{s l}-\frac{x_{s l}}{\mu_{0} A_{s l}^{a r l}}\right) \mu_{0} A_{s l}^{a r m}} \\
+ & \frac{\left(\mathcal{R}_{s l}-\frac{x_{s l}}{\mu_{0} A_{s l}^{a r m}}\right)\left(U_{s u p}-R_{s l} i_{s l}-u_{s l}^{p w s}\right)}{N_{s l}{ }^{2}} \\
f_{2, \mathcal{M}_{0}}^{(k)}= & \frac{2\left(\mathcal{R}_{s l}-\frac{x_{s l}}{\mu_{0} A_{s l}^{a r m}}\right)^{2}}{m_{s l}} \frac{1}{\mu_{0} A_{s l}^{a r m}} \\
& -\frac{s_{s l}\left(x_{s l}+x_{s l, 0}\right)+k_{s l} v_{s l}+F_{s l}^{l i m}}{m_{s l}}
\end{aligned}
$$

$$
f_{3, \mathcal{M}_{0}}^{(k)}=v_{s l}
$$

$$
\begin{aligned}
f_{4, \mathcal{M}_{0}}^{(k)}= & -\frac{v_{b l} i_{b l}}{\left(\mathcal{R}_{b l}-\frac{x_{b l}}{\mu_{0} A_{b l}^{a r m}}\right) \mu_{0} A_{b l}^{a r m}} \\
& +\frac{\left(\mathcal{R}_{b l}-\frac{x_{b l}}{\mu_{0} A_{b l}^{a m m}}\right)\left(U_{s u p}-R_{b l} i_{b l}-u_{b l}^{p w s}\right)}{N_{b l}{ }^{2}}
\end{aligned}
$$

$$
\begin{gathered}
f_{5, \mathcal{M}_{0}}^{(k)}=\frac{\frac{\left(N_{b l} i_{b l}\right)^{2}}{2\left(\mathcal{R}_{b l}-\frac{x_{b l}}{\mu_{0} A_{b l}^{a m}}\right)^{2}} \frac{1}{m_{b l}}}{\mu_{0 l}^{a r m}} \\
-\frac{s_{b l}\left(x_{b l}+x_{b l, 0}\right)+k_{b l} v_{b l}+F_{b l}^{l i m}}{m_{b l}} \\
f_{6, \mathcal{M}_{0}}^{(k)}=v_{b l}
\end{gathered}
$$

$$
\begin{aligned}
f_{7, \mathcal{M}_{0}}^{(k)}= & -\frac{v_{s e} i_{s e}}{\left(\mathcal{R}_{s e}-\frac{x_{s e}}{\mu_{0} A_{s e}^{a r m}}\right) \mu_{0} A_{s e}^{\text {arm }}} \\
& +\frac{\left(\mathcal{R}_{s e}-\frac{x_{s e}}{\mu_{0} A_{s e}^{a r m}}\right)\left(U_{s u p}-R_{s e} i_{s e}-u_{s e}^{p w s}\right)}{N_{s e}{ }^{2}}
\end{aligned}
$$

$$
\begin{aligned}
f_{10, \mathcal{M}_{0}}^{(k)}= & -\frac{v_{b e} i_{b e}}{\left(\mathcal{R}_{b e}-\frac{x_{b e}}{\mu_{0} A_{b e}^{a r e}}\right) \mu_{0} A_{b e}^{a r m}} \\
& +\frac{\left(\mathcal{R}_{b e}-\frac{x_{b e}}{\mu_{0} A_{b e}^{a m}}\right)\left(U_{s u p}-R_{b e} i_{b e}-u_{b e}^{p w s}\right)}{N_{b e}{ }^{2}}
\end{aligned}
$$

$$
\begin{gathered}
f_{11, \mathcal{M}_{0}}^{(k)}=\frac{\frac{\left(N_{b e} i_{b e}\right)^{2}}{\left(\mathcal{R}_{b e}-\frac{x_{b e}}{\mu_{0} A_{b e}^{a r m}}\right)^{2} \frac{1}{\mu_{0} A_{b e}^{a r m}}}}{m_{b e}} \\
-\frac{s_{b e}\left(x_{b e}+x_{b e, 0}\right)+k_{b e} v_{b e}+F_{b e}^{l i m}}{m_{b e}} \\
f_{12, \mathcal{M}_{0}}^{(k)}=v_{b e}
\end{gathered}
$$

$$
\begin{aligned}
f_{13, \mathcal{M}_{0}}^{(k)} & =\left(\alpha_{s l} A_{s l}+\alpha_{b l} A_{b l}\right) \xi_{l} \\
& -\left(\alpha_{s e} A_{s e}+\alpha_{b e} A_{b e}\right) \xi_{e}
\end{aligned}
$$




$$
\begin{aligned}
& f_{14, \mathcal{M}_{0}}^{(k)}= \frac{\kappa \mathrm{R}\left(\alpha_{s l} A_{s l}+\alpha_{b l} A_{b l}\right) \xi_{l} T_{s u p}}{V_{c h}^{d}+x_{p s t} A_{p s t}} \\
&-\frac{\kappa p_{c h}\left(\alpha_{s e} A_{s e}+\alpha_{b e} A_{v e}\right) \xi_{e}}{m_{c h}} \\
&-\frac{(\kappa-1) \mathrm{k}_{h t} A_{h t}\left(\frac{p_{c h}\left(V_{c h}^{d}+x_{p s t} A_{p s t}\right)}{m_{c h} \mathrm{R}}-T_{a m b}\right)}{V_{c h}^{d}+x_{p s t} A_{p s t}} \\
&-\frac{\kappa p_{c h} v_{p s t} A_{p s t}}{V_{c h}^{d}+x_{p s t} A_{p s t}} \\
& f_{15, \mathcal{M}_{0}}^{(k)} \frac{\left(p_{c h}-p_{a m b}\right) A_{p s t}+s_{h s p}\left(x_{h s p_{0}}-x_{p s t}\right)}{m_{p s t}} \\
&-\frac{F_{f r}+v_{p s t} k_{p s t}+F_{p s t}^{l i m}+F_{l}\left(x_{p s t}\right)}{m_{p s t}} \\
& f_{16, \mathcal{M}_{0}}^{(k)}=v_{p s t}
\end{aligned}
$$

where

$$
\begin{gathered}
=\sqrt{\frac{1}{1 \mathrm{R}_{\text {sup }}}\left(\Pi_{l}^{\frac{2}{\kappa}}-\Pi_{l}{ }_{l}^{\frac{1}{\kappa}}\right)} \\
\xi_{e}=\sqrt{\frac{2 \kappa}{\kappa-1} \frac{p_{c h} m_{c h}}{\left(V_{c h}^{d}+x_{p s t} A_{p s t}\right)}\left(\Pi_{e}^{\frac{2}{\kappa}}-\Pi_{e}^{\frac{\kappa+1}{\kappa}}\right)}
\end{gathered}
$$

The model includes five subsystem types that exhibit discrete-continuous behavior. The first is the voltage drop of the power stages $\left(u_{x x}^{p w s}\right)$ for the four SMVs:

$$
u_{x x}^{p w s}=\left\{\begin{array}{cl}
U_{s u p}\left(1-m_{d, x x}\right) & m_{d, x x}>0 \\
+r_{D S(o n)} i & m_{d, x x}=0 \text { and } i_{x x}>0 \\
U_{B R} & m_{d, x x}=0 \text { and } i_{x x}=0 \\
U_{s u p} &
\end{array}\right.
$$

The next are the armature stroke limiting force $\left(F_{x x}^{l i m}\right)$ and flow cross-section $\left(A_{x x}\right)$ of the SMVs, which are dependent terms for the same SMV. Hence, these two terms together give four independent modes for each SMV:

$$
F_{x x}^{l i m}=\left\{\begin{array}{cc}
c_{1, x x}\left(x_{x x}-x_{x x}^{l i m, 1}\right) & x_{x x}<x_{x x}^{l i m, 1} \\
+k_{1, x x} v_{x x} & x_{x x}^{l i m, 1} \leq x_{x x}<x_{x x}^{l i m, 2} \\
0 & x_{x x}^{l i m, 2} \leq x_{x x} \\
c_{2, x x}\left(x_{x x}-x_{x x}^{l i m, 2}\right) & \\
+k_{2, x x} v_{x x} &
\end{array}\right.
$$

$$
A_{x x}=\left\{\begin{array}{cc}
0 & x_{x x}<x_{x x}^{l i m, 1} \\
x_{x x} d_{x x} \pi & x_{x x}^{l i m, 1} \leq x_{x x}<\frac{d_{x x}}{4} \\
\frac{d_{x x}^{2}}{4} \pi & \frac{d_{x x}}{4} \leq x_{x x}
\end{array}\right.
$$

The third are the air flow terms for load $\left(\prod_{l}\right)$ and exhaust $\left(\prod_{e}\right)$ directions:

$$
\begin{gathered}
\Pi_{l}=\left\{\begin{array}{cc}
\frac{p_{c h}}{p_{\text {sup }}} & 1 \geq \frac{p_{c h}}{p_{\text {sup }}}>\Pi_{c r i t} \\
\Pi_{c r i t} & \frac{p_{c h}}{p_{\text {sup }}} \leq \Pi_{\text {crit }}
\end{array}\right. \\
\Pi_{e}=\left\{\begin{array}{cc}
\frac{p_{\text {amb }}}{p_{c h}} & 1 \geq \frac{p_{a m b}}{p_{c h}}>\Pi_{c r i t} \\
\Pi_{c r i t} & \frac{p_{a m b}}{p_{c h}} \leq \Pi_{c r i t}
\end{array}\right.
\end{gathered}
$$

where

$$
\Pi_{\text {crit }}=\left(\frac{2}{\kappa+1}\right)^{\frac{\kappa}{\kappa-1}}
$$

is the critical pressure ratio.

The fourth term is the piston stroke limiting force $\left(F_{p s t}^{\lim }\right)$ :

$$
F_{p s t}^{l i m}=\left\{\begin{array}{cc}
c_{p s t}\left(x_{p s t}-x_{p s t}^{l i m, 1}\right)+ & x_{p s t}<x_{p s t}^{l i m, 1} \\
+k_{p s t} v_{p s t} & x_{p s t}^{l i m, 1} \leq x_{p s t}<x_{p s t}^{l i m, 2} \\
0 & x_{p s t}^{l i m, 2} \leq x_{p s t} \\
c_{p s t}\left(x_{p s t}-x_{p s t}^{l i m, 2}\right)+ & \\
+k_{p s t} v_{p s t} &
\end{array}\right.
$$

Finally the last is the friction force of the piston $\left(F_{f r}\right)$ :

$$
F_{f r}=\left\{\begin{array}{cc}
-\mu_{p s t}\left(p_{c h}-p_{a m b}\right) A_{p s t} & v_{p s t}<0 \\
0 & v_{p s t}=0 \\
\mu_{p s t}\left(p_{c h}-p_{a m b}\right) A_{p s t} & v_{p s t}>0
\end{array}\right.
$$

Since the output is linear with respect to the state vector, the measured output is written as the following equation:

$$
\mathbf{y}_{\mathcal{M}_{0}}=\mathbf{C} \mathbf{x}_{\mathcal{M}_{0}}
$$

where the $\mathbf{C}$ matrix is determined by the intended application of the model e.g., on purpose to do simulations for verification $\mathbf{C}$ can be equal to the identity matrix to reach all the state variables. As opposed to the simulation the instrumental conditions do not make it possible to reach all the state variables thus the model output matrix has been selected as follows: $\mathbf{C}=\left\langle c_{i, j}\right\rangle$, here $c_{1,14}=c_{2,16}=1$ and the remained elements are equal to zero. 
In this way the model outputs are the chamber pressure $\left(p_{c h}\right)$ and the piston position $\left(x_{p s t}\right)$.

The values of disturbance inputs and parameters, considered in the simulation calculations, are shown in Table 1 and Table 2 respectively.

Table 1 List of disturbance inputs

\begin{tabular}{cccc}
\hline Name & Symbol & Value & Unit \\
\hline Supply voltage & $U_{\text {sup }}$ & 24 & $\mathrm{~V}$ \\
\hline Supply pressure & $p_{\text {sup }}$ & $9,5 \times 10^{5}$ & $\mathrm{~Pa}$ \\
\hline Ambient pressure & $p_{\text {amb }}$ & $1 \times 10^{5}$ & $\mathrm{~Pa}$ \\
\hline Supply temperature & $T_{\text {sup }}$ & 293 & $\mathrm{~K}$ \\
\hline Ambient temperature & $T_{a m b}$ & 293 & $\mathrm{~K}$ \\
\hline
\end{tabular}

The verification of this model has been carried out by using extensive simulations against engineering perception and operational experience on the qualitative behavior. For validation purposes the outputs of the model have been compared to measurements on the real system to give a quantitative performance index about the model accuracy. Then, it has been found that this model has been able to predict the dynamic behavior of the EPC system within the accuracy required for dynamic simulation, and control validation purposes (Szimandl, Németh, 2013).

Before attempting to simplify a dynamic model, the hierarchy diagram and the variable structure of the model should be examined. The model elements can be organized into the following hierarchical levels to get the hierarchy diagram of the model (see details in Hangos, Cameron (2001)): balance volume level (top), balance equation level, terms in balance equations corresponding to the mechanisms, constitutive equations and parameters (bottom).

The hierarchy diagram of the detailed EPC model is shown in Fig. 2 where the lower levels of the hierarchy are joined together. In the balance volume level ten balance volumes are found; one for each SMV armature, one for each SMV solenoid winding, one for the clutch chamber and one for the piston. The armature and solenoid balance volumes correspond to four valves hence; these terms and the corresponding ones are encircled by a dotted line and marked with $\times 4$. In the balance equation level, which regards to mass, energy and momentum balances, 16 state variables are presented. In the lowest level the transport mechanisms and the related parameters are collected. The simulations show that the momentum and energy balances, correspond to armature and solenoid balance volumes (light red terms), have fast dynamics meanwhile, the heat transfer term, which corresponds to the energy balance of the chamber (light blue term), has slow dynamics comparing with the remained terms (light green). The mechanisms, which introduce hybrid behavior, are depicted by dashed lines.

The variable structure of a model can be represented with directed graph description (see the details in Hangos, Cameron (2001)). In the variable structure graph of the EPC model (see Fig. 3) the variables are in circles. Four of the boxed states are related to the four power stage / valves subsystem and the remained one for the chamber / piston subsystem.

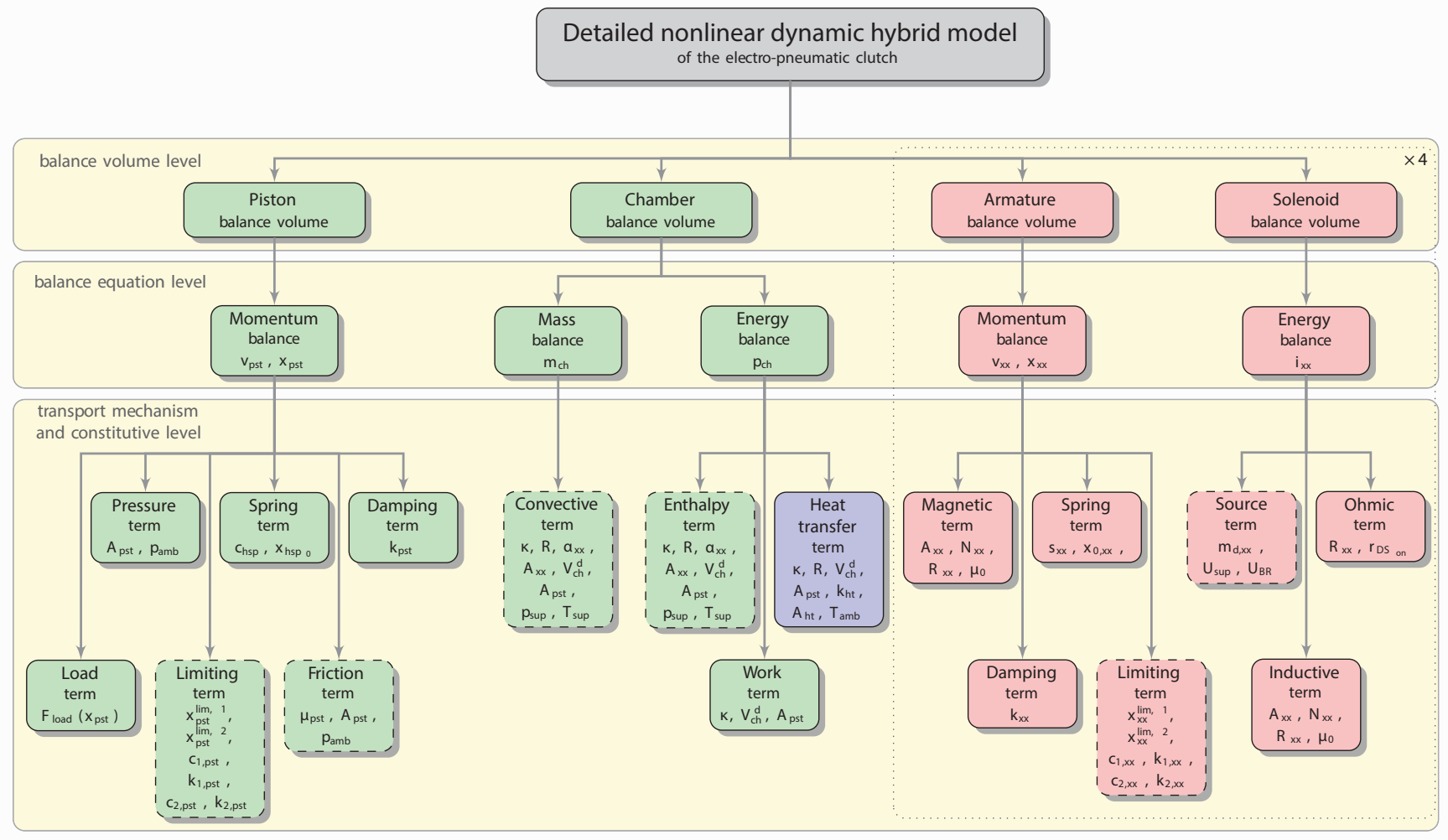

Fig. 2 Hierarchical structure of the detailed nonlinear dynamic hybrid model 
Table 2 List of parameters

\begin{tabular}{|c|c|c|c|}
\hline Name & Symbol & Value & Unit \\
\hline Adiabatic exponent & $\kappa$ & 1.4 & - \\
\hline Permeability of vacuum & $\mu_{0}$ & $4 \pi \times 10^{-7}$ & $\mathrm{Vs} / \mathrm{Am}$ \\
\hline Specific gas constant & $\mathrm{R}$ & 287.14 & $\mathrm{~J} / \mathrm{kgK}$ \\
\hline Drain to source on resistance & $r_{D S(o n)}$ & 0.071 & $\Omega$ \\
\hline Effective breakdown voltage & $U_{B R}$ & 71 & $\mathrm{~V}$ \\
\hline Inlet diameter of small SMVs & $d_{s x}$ & 0.0015 & $\mathrm{~m}$ \\
\hline Inlet diameter of big SMVs & $d_{b x}$ & 0.0035 & $\mathrm{~m}$ \\
\hline Armature diameter of small SMVs & $d_{a r m, s x}$ & 0.010 & $\mathrm{~m}$ \\
\hline Armature diameter of big SMVs & $d_{a r m, b x}$ & 0.012 & $\mathrm{~m}$ \\
\hline Return spring stiffness of small SMVs & $s_{s x}$ & 489 & $\mathrm{~N} / \mathrm{m}$ \\
\hline Return spring stiffness of big SMVs & $s_{b x}$ & 567 & $\mathrm{~N} / \mathrm{m}$ \\
\hline Return spring pretension of small SMVs & $x_{s x, 0}$ & 0.0102 & $\mathrm{~m}$ \\
\hline Return spring pretension & $x_{b x, 0}$ & 0.0063 & $\mathrm{~m}$ \\
\hline Position of SMVs seat of big SMVs & $x_{x x}^{\text {lim }, 1}$ & 0 & $\mathrm{~m}$ \\
\hline Stroke limitation of small SMVs & $x_{s x}^{l i m, 2}$ & 0.0009 & $\mathrm{~m}$ \\
\hline Stroke limitation of big SMVs & $x_{b x}^{\lim , 2}$ & 0.0012 & $\mathrm{~m}$ \\
\hline Armature mass of small SMVs & $m_{s x}$ & 0.012 & $\mathrm{~kg}$ \\
\hline Armature mass of big SMVs & $m_{b x}$ & 0.016 & $\mathrm{~kg}$ \\
\hline Solenoid turns of small SMVs & $N_{s x}$ & 600 & - \\
\hline Solenoid turns of big SMVs & $N_{b x}$ & 910 & - \\
\hline Solenoid resistance of small SMVs & $R_{s x}$ & 11.24 & $\Omega$ \\
\hline Solenoid resistance of big SMVs & $R_{b x}$ & 9.339 & $\Omega$ \\
\hline Solenoid reluctance of SMVs & $R_{x x}$ & $1.2 \mathrm{e} 7$ & $\mathrm{~A} / \mathrm{Vs}$ \\
\hline Contraction coefficient of SMVs & $\alpha_{x x}$ & 0.63 & - \\
\hline Damping coefficient of SMVs & $k_{x x}$ & 30 & $\mathrm{Ns} / \mathrm{m}$ \\
\hline Seat stiffness of SMVs & $c_{1, x x}$ & $10^{7}$ & $\mathrm{~N} / \mathrm{m}$ \\
\hline Stroke limitation stiffness & $c_{2, x x}$ & $10^{7}$ & $\mathrm{~N} / \mathrm{m}$ \\
\hline Seat damping of SMVs of SMVs & $k_{l, x x}$ & $10^{7}$ & $\mathrm{Ns} / \mathrm{m}$ \\
\hline Stroke limitation damping & $k_{2, x x}$ & $10^{7}$ & $\mathrm{Ns} / \mathrm{m}$ \\
\hline Stiffness of helper spring of SMVs & $s_{h s p}$ & $1 \times 10^{4}$ & $\mathrm{~N} / \mathrm{m}$ \\
\hline Pretension stroke of helper spring & $x_{h s p_{0}}$ & 0.06 & $\mathrm{~m}$ \\
\hline Idle stroke of disc spring & $x_{d s p_{0}}$ & $0,33 \times 10^{-3}$ & $\mathrm{~m}$ \\
\hline Area of piston & $A_{p s t}$ & 0.0227 & $\mathrm{~m}^{2}$ \\
\hline Dead volume of chamber & $V_{c h}^{d}$ & $5,59 \times 10^{-4}$ & $\mathrm{~m}^{3}$ \\
\hline Lumped mass & $m_{p s t}$ & 9.4 & $\mathrm{~kg}$ \\
\hline Heat transfer coefficient of chamber & $k_{h t}$ & 0.02 & $\mathrm{~W} / \mathrm{m}^{2} \mathrm{~K}$ \\
\hline Heat transfer area of chamber & $A_{h t}$ & 0.07 & $\mathrm{~m}^{2}$ \\
\hline Damping coefficient of piston & $k_{p s t}$ & 2250 & $\mathrm{Ns} / \mathrm{m}$ \\
\hline Friction coefficient of piston & $\mu_{p s t}$ & 0.135 & - \\
\hline Position of piston stroke limitation 1 & $x_{p s t}^{\text {lim, } 1}$ & -0.005 & $\mathrm{~m}$ \\
\hline Position of piston stroke limitation 2 & $x_{p s t}^{\text {lim, }}$ & 0.030 & $\mathrm{~m}$ \\
\hline Stroke limitation 1 stiffness of piston & $c_{1, p s t}$ & $10^{8}$ & $\mathrm{~N} / \mathrm{m}$ \\
\hline Stroke limitation 2 stiffness of piston & $c_{2, p s t}$ & $10^{8}$ & $\mathrm{~N} / \mathrm{m}$ \\
\hline Stroke limitation 1 damping of piston & $k_{l, p s t}$ & $10^{8}$ & $\mathrm{Ns} / \mathrm{m}$ \\
\hline Stroke limitation 2 damping of piston & $k_{2, p s t}$ & $10^{8}$ & $\mathrm{Ns} / \mathrm{m}$ \\
\hline
\end{tabular}




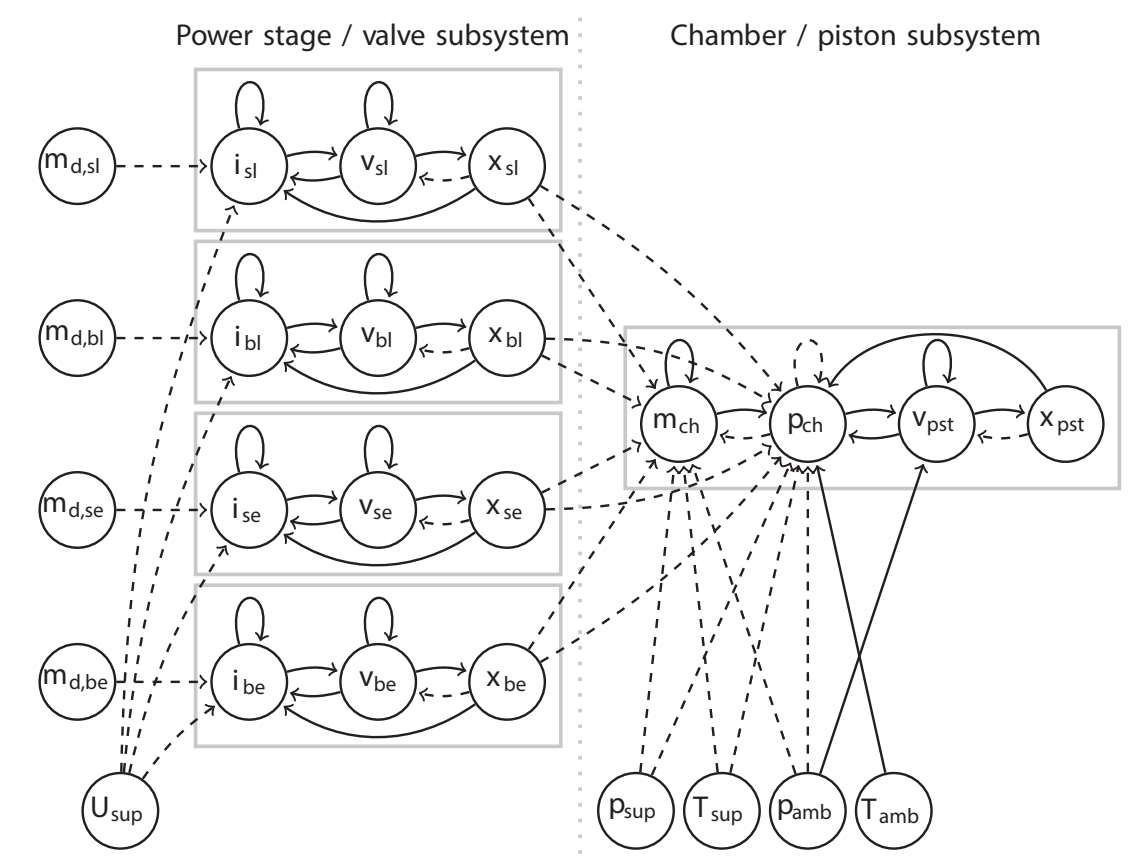

Fig. 3 Structure graph of the differential variables of the EPC model

The four input variables are on left side and the disturbance inputs are located at bottom. The edges that depend on hybrid terms are depicted by dashed lines as well. The total number of the hybrid modes can be calculated by the combination of the independent ones as: $n_{r}=3^{4} \times 4^{4} \times 2 \times 2 \times 3 \times 3=746496$.

\section{Model simplification procedure}

The model simplification is performed by applying model simplification assumptions to the detailed model to reduce its size and complexity, where the model elements to be left out or lumping together are selected based on engineering judgment and physical insights. The application of the simplifying assumptions is repeated meanwhile the previously specified model simplifying goals are satisfied. The flowchart of the model simplification procedure is shown in Fig. 4.

The first step of the model simplification process is to specify the model simplifying goals. The modeling goals, i.e. the set of performance indices, are specified by the intended use of the model. Hence the modeling goal set is a complex statement and it to be given in terms of a set of performance indices $\left[\chi_{1}\right.$ $\left.\ldots \chi_{n}\right]$. The performance index $\chi_{i}$ can be real and/or Boolean quantity which defined for the model $M$ as: $\chi_{i}: M \rightarrow \mathbb{R}, \mathbb{B}$. In this instance the performance index represents a model characteristic that is captured as a real and/or Boolean valued quantity e.g., model accuracy, hybrid behavior, presence of nonlinearity and so on. Note that the Boolean items can express the presence or absence of a characteristic. Furthermore, each real valued performance index can be stated with acceptance limits in the form of inequalities: $\aleph_{u i}^{\min } \leq \leq{ }^{\max }, \ddot{u}=1, \quad$, .

If different models are being compared with respect to their simplicity, a suitable quality or size norm that reflects simplicity should be defined as well. In case of size indices, it can be

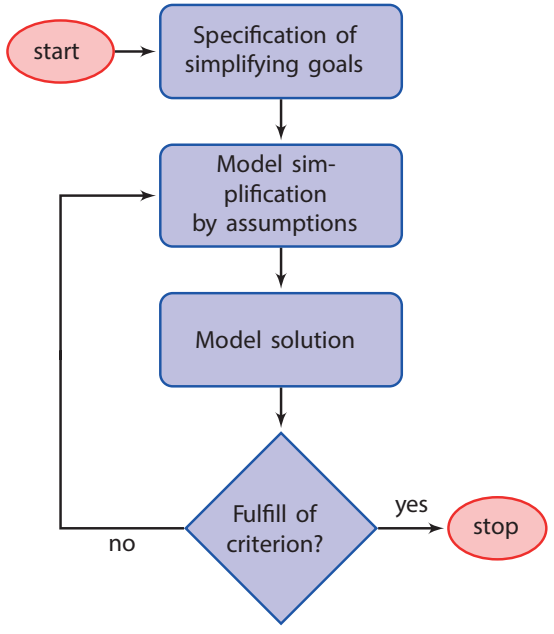

Fig. 4 Model simplification procedure

defined integer-valued numbers, which characterize the size of a model in a given sense. Thus, a size index $\xi_{i}$ is defined as: $\xi_{i}: M \rightarrow \mathbb{Z}$. In this context size refers to an inherent feature or property of the model being considered hence, it could refer to such model features as state variable dimension, number of the parameters and hybrid modes, nonlinearity measure and so on. Given a set of size indices $\xi_{1}, \ldots, \xi_{m}$ on a model a vector $\xi \in \mathbb{Z}^{m}$ can be formed with the size index values as its entries, such that: $\xi=\left[\xi_{1}, \ldots, \xi_{m}\right]^{T}$. Then, the size norm is defined as a vector norm on the vector space $\mathbb{Z}^{m}$ with the usual Euclidean vector norm as:

$$
\|\xi\|=\sqrt{\xi_{1}^{2}+\ldots+\xi_{m}^{2}}
$$

When two different models are being compared by using a size index depending upon a model element then it may happen that such an element is simply not present in at least one of the 
models. Formally it means that the value of this element is nonexisting. Therefore, every size index should be defined in such a way that it gives zero for a non-existing element.

In the beginning of the simplification many possible assumptions can be done, but the most influential and the independent ones should be applied first to achieve systematic model reduction instead of heuristic. Since, if one makes a simplification assumption to any of the model elements that will influence all the other elements on the lower level(s) that are related to it. Hence, leaving out balance volume(s) from the model should be tried firstly and then leaving out the unimportant balance equations and/or mechanisms and finally variable/parameter lumping should be considered.

In the next step the output response of the simplified model is derived by solving the simplified set of DAEs and the simplified model performance indices are determined accordingly. The test cases to be used are determined based on the desired use of the model and its operation domain covering the most important dynamic behavior the system is able to produce.

Finally, in the decision making on one hand the model performance indices and on the other hand the size indices are compared to the specified ones to decide whether the model fulfill the simplifying goals or not. If all the goals are fulfilled the simplifying procedure is terminated otherwise jumps back to make further investigation on the model behavior to apply further assumptions or revise the assumptions that previously done to apply better ones.

\section{Simplification results}

In general, the simplified model should retain the major dynamic characteristics of the system only and omit all the details that are weakly represented in the outputs where the major dynamic characteristics are depend on the intended use of the model.

\subsection{Simplified nonlinear dynamic model for simulation purposes $\left(\mathcal{M}_{1}\right)$}

The following properties, defined by performance $\left(\mathcal{P}_{x}\right)$ and size $\left(\mathcal{S}_{x}\right)$ indices, of the simplified EPC model $\left(\mathcal{M}_{1}\right)$ are considered below to achieve the simplifying goals, which are in this case fast dynamic simulation with reduced computational effort to make it possible to integrate the EPC model into a complete driveline or a vehicle model.

$\mathcal{M}_{1} \mathcal{P}_{1}$ The model variables and parameters should preserve the physical meaning $\left(\chi_{\mathcal{M}_{1} \mathcal{P}_{1}} \in \mathbb{B}\right)$.

$\mathcal{M}_{1} \mathcal{P}_{2}$ The model should be capable of describing the dynamic behavior of the EPC system within 5\% deviation in the whole operation domain with respect to the chamber pressure and the piston position $\left(\chi_{\mathcal{M}_{1} \mathcal{P}_{2}} \in \mathbb{R}, \chi_{\mathcal{M}_{1} \mathcal{P}_{2}}^{\max }=0.05\right)$. This performance index is calculated by the Euclidian norm as:

$$
\chi_{\mathcal{M}_{1} \mathcal{P}_{2}}=\sqrt{\chi_{1, \mathcal{M}_{1} \mathcal{P}_{2}}^{2}+\chi_{2, \mathcal{M}_{1} \mathcal{P}_{2}}^{2}}
$$

where $\chi_{1 \mathcal{M}_{1} \mathcal{P}_{2}}$ is the chamber pressure deviation and $\chi_{2 \mathcal{M}_{1} \mathcal{P}_{2}}$ is the piston position deviation. For computing $\chi_{i \mathcal{M}_{1} \mathcal{P}_{2}}$ an $L_{2}$ error has been used to measure the deviations of the model response, based on the entries of the model output vectors and the measurement results on the real system as follows:

$$
\chi_{i, \mathcal{M}_{1} \mathcal{P}_{2}}=\sqrt{\frac{1}{T} \int_{0}^{T}\left(\frac{y_{i}^{\text {Meas }}(t)-y_{i}^{\mathcal{M}_{1}}(t)}{\bar{y}_{i}^{\text {Meas }}}\right)^{2} d t}
$$

where the suffix $i$ refers to the $i$ th output of the model, Meas and $\mathcal{M}_{1}$ refer to the corresponding output vector of the measurement and simulation respectively. The over line refers to the integral mean of the particular signal and $T$ is the duration of the test case.

$\mathcal{M}_{1} \mathcal{S}_{1}$ The model size and complexity should be significantly reduced $\left(\xi_{\mathcal{M}_{1} \mathcal{S}_{1}} \in \mathbb{R}\right)$. The size index is also calculated by the Euclidian norm as:

$$
\xi_{\mathcal{M}_{1} \mathcal{S}_{1}}=\sqrt{w_{1} \xi_{1, \mathcal{M}_{1} \mathcal{S}_{1}}^{2}+\ldots+\omega_{m} \xi_{m, \mathcal{M}_{1} \mathcal{S}_{1}}^{2}}
$$

where $\xi_{1, \mathcal{M}_{1} \mathcal{S}_{1}} \ldots \xi_{m, \mathcal{M}_{1} \mathcal{S}_{1}}$ are the number of the balance volumes, state variables, control and disturbance inputs, parameters, hybrid terms, relative degree and finally the relative computational time of the simulated time stretch, i.e. the calculation time divided by the simulated time. Moreover, in order to achieve the weighted sum of the individual squared numbers $w_{i}$ weights are used.

To achieve the specified goals above via systematic model simplification the first step is to simplifying the balance volumes then the balance equations and finally the transport mechanisms and constitutive equations. Hence, the following assumptions are done:

$\mathcal{M}_{1} \mathcal{A}_{1}$ Remove the power stage / valve subsystem dynamics from the model and apply ideal valves in which the opening and closing processes are instantaneous.

This assumption can be done since the power stage / valve subsystem has much faster dynamics than the remained model parts and there is not any feedback therefrom. It removes the $i_{x x}, v_{x x}$ and $x_{x x}$ terms from the state vector, the $U_{\text {sup }}$ term from the disturbance inputs besides, removes all the parameters that related to the power stage / valve subsystem except $\alpha_{x x}$ and $A_{x x}$.

$\mathcal{M}_{1} \mathcal{A}_{2}$ The thermodynamic processes in the chamber can be considered as isothermal instead of polytropic. 
This assumption can be done as well since the focused dynamical range of the modeled processes should cover the medium dynamic responses only while the air temperature converges to the environment temperature with slow dynamics. In this way the temperature in the chamber can be considered with constant value. Thus, the isothermal model class can cover the required behavior. One has to mention that this assumption eliminates the $m_{c h}$ state since the chamber balance equation is derived from the gas mass balance only and the gas energy balance equation is not needed anymore. Moreover, eliminates the $T_{\text {sup }}$ disturbance input by using $T_{a m b}$.

\section{$\mathcal{M}_{1} \mathcal{A}_{3}$ The piston cannot reach its limitations during nor- mal operation.}

One of the extreme piston positions, corresponding of the completely exhausted chamber, is determined by the stiffness of the disc and helper springs without reaching the stroke limitation $\left(x_{p s t}^{l i m, 1}\right)$. Besides, the piston stroke is large enough to cover the working domain of the clutch mechanism without reaching the other stroke limitation $\left(x_{p s t}^{\text {lim, } 2}\right)$. This assumption results that the piston limiting term $\left(F_{p s t}^{\text {lim }}\right)$ can be left out of the model, which reduces the number of the hybrid modes and eliminates the computational effort regarding the collisions with the stroke limiters.

$\mathcal{M}_{1} \mathcal{A}_{4}$ Subsonic flow in both of the intake and exhaust direction cannot reached simultaneously.

The necessary condition for achieving subsonic flow in both of the two direction, when $\prod_{l}>\prod_{\text {crit }}$ and $\prod_{e}>\prod_{\text {crit }}$, is $p_{\text {amb }} / p_{\text {sup }}>\Pi_{\text {crit }}^{2}$. But this condition has not been satisfied since it is out of the (disturbance) input constraints of the EPC (Szimandl, Németh, 2013).

$\mathcal{M}_{1} \mathcal{A}_{5}$ The friction force term $\left(F_{f r}\right)$ can be approximated using sigmoid function as follows:

$$
F_{f r}=\mu_{p s t}\left(p_{c h}-p_{a m b}\right) A_{p s t}\left(\frac{2}{1-e^{-u_{f r} v_{p s t}}}-1\right)
$$

where $u_{f r}$ determines the friction force steepness at small velocities. This improves the numeric problems eliminating the hybrid modes regarding the friction force.

\section{$\mathcal{M}_{1} \mathcal{A}_{6}$ The cross sections and the contraction coefficients can be lump together.}

The new, i.e. effective, cross section terms are obtained as follows: $A_{x x}^{\text {eff }}=\alpha_{x x} A_{x x}$.
$\mathcal{M}_{1} \mathcal{A}_{7}$ For the sake of simplicity, the spring term $s_{h s p}\left(x_{p s t, 0}\right.$ $\left.-x_{p s t}\right)$ should be integrated into the $F_{l}\left(x_{p s t}\right)$ term.

Applying these assumptions above the state space description of the simplified model for simulation purposes is obtained. From the simplified DAEs of the model the state vector is composed as follows:

$$
\mathbf{x}_{\mathcal{M}_{1}}=\left[\begin{array}{lll}
p_{c h} & v_{p s t} & x_{p s t}
\end{array}\right]^{T}
$$

The control input vector $\left(\mathbf{u}_{\mathcal{M}_{1}}\right)$, includes the duty cycle of the PWM control signals of the SMVs, is not changed (see Eq. (2)), while the disturbance input vector includes only the compressed air pressure, ambient- pressure and temperature respectively:

$$
\mathbf{d}_{\mathcal{M}_{1}}=\left[\begin{array}{lll}
p_{\text {sup }} & p_{\text {amb }} & T_{\text {amb }}
\end{array}\right]^{T}
$$

The simplified state space model has the form of Eq. (4) as well where the nonlinear state functions are written as follows (the entries that depend on the hybrid modes are boxed also):

$$
\begin{aligned}
f_{1, \mathcal{M}_{1}}^{(k)}= & \frac{\mathrm{R}\left(m_{d, s l} A_{s l}^{e f f}+m_{d, b l} A_{b l}^{e f f}\right) \xi_{l} T_{a m b}}{V_{c h}^{d}+x_{p s t} A_{p s t}} \\
& -\frac{\mathrm{R}\left(m_{d, s e} A_{s e}^{e f f}+m_{d, b e} A_{b e}^{e f f}\right) \xi_{e} T_{a m b}}{V_{c h}^{d}+x_{p s t} A_{p s t}} \\
& -\frac{p_{c h} v_{p s t} A_{p s t}}{V_{c h}^{d}+x_{p s t} A_{p s t}}
\end{aligned}
$$

$$
\begin{aligned}
f_{2, \mathcal{M}_{1}}^{(k)}= & \frac{\left(p_{c h}-p_{a m b}\right) A_{p s t}-v_{p s t} k_{p s t}-F_{l}\left(x_{p s t}\right)}{m_{p s t}} \\
& -\frac{\mu_{p s t}\left(p_{c h}-p_{a m b}\right) A_{p s t}\left(\frac{2}{1-e^{-u f f} v_{p s t}}-1\right)}{m_{p s t}}
\end{aligned}
$$

$$
f_{3, \mathcal{M}_{1}}^{(k)}=v_{p s t}
$$

where

$$
\xi_{l}=\sqrt{\frac{2 \kappa}{\kappa-1} \frac{p_{s u p}^{2}}{\mathrm{R} T_{a m b}}\left(\Pi_{l}^{\frac{2}{\kappa}}-\Pi_{l}^{\frac{\kappa+1}{\kappa}}\right)}
$$

$$
\xi_{e}=\sqrt{\frac{2 \kappa}{\kappa-1} \frac{p_{c h}^{2}}{\mathrm{R} T_{a m b}}\left(\Pi_{\left.e^{\frac{2}{\kappa}}-\Pi_{e}^{\frac{\kappa+1}{\kappa}}\right)}\right.}
$$

The simulation result of the simplified $\operatorname{model}\left(\mathcal{M}_{1}\right)$ is shown in Fig. 5. Besides, the measurement on the real system and the response of the detailed model $\left(\mathcal{M}_{0}\right)$ for the same inputs are shown as well. The input signals are derived from a real clutching. The disengagement has been executed with maximal dynamic to reach the disengagement state as short as possible. Then, the engagement has been started with high dynamic as well to reach 


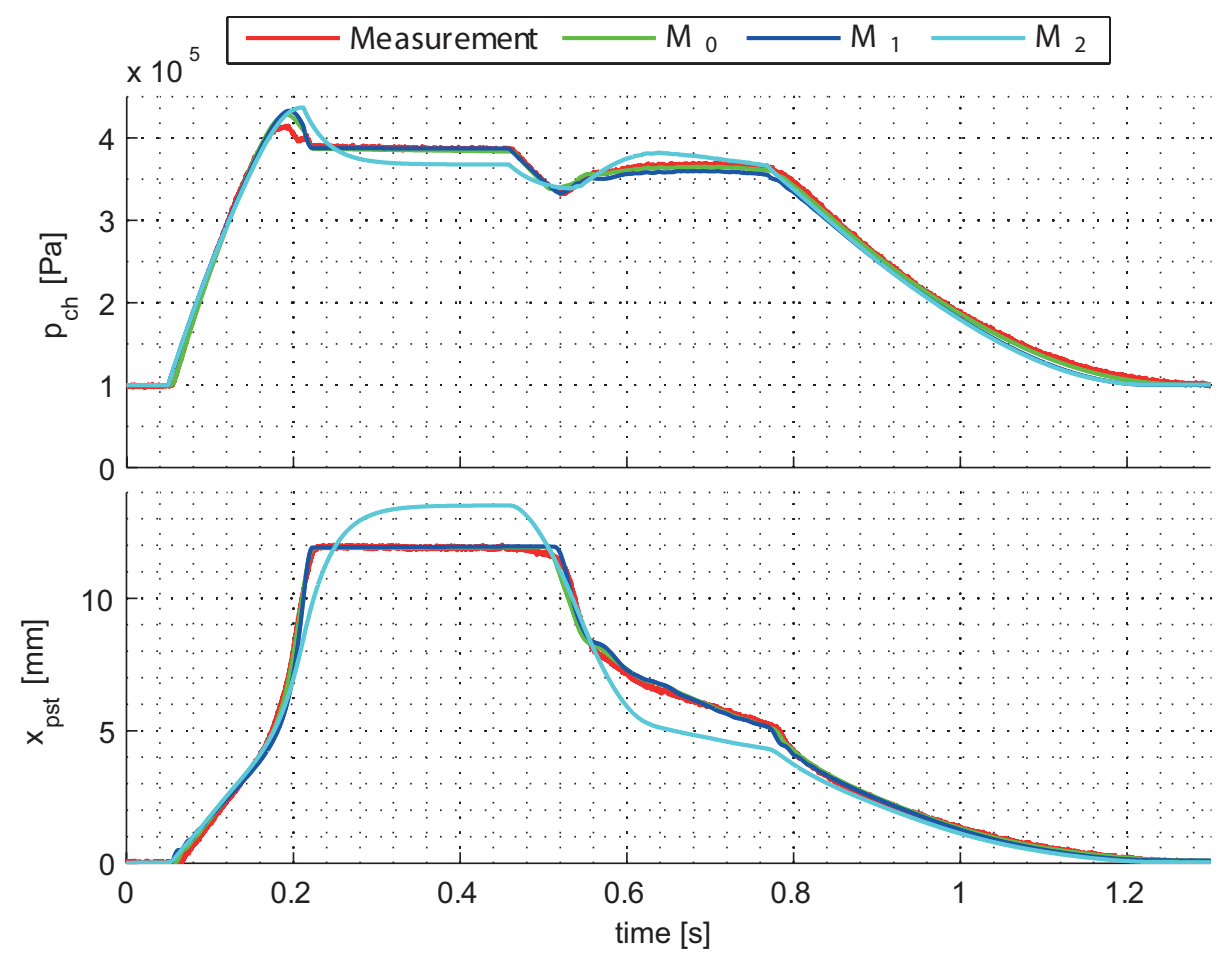

Fig. 5 EPC measurement and simulation results

the touch point, where the pressure plate and the friction disc have been connected. In the clutching domain the stroke has been decreased slowly to increase the transmitted torque smoothly. Finally, in the end of the engagement the engagement state has been set up with maximal dynamic as well.

All the retained system variables have preserved their physical meaning meanwhile, some model parameters $\left(\alpha_{x x}\right.$ and $A_{x x}$ ) have changed slightly their meaning due to the lumping $\left(\chi_{\mathcal{M}_{1} p_{1}}=\right.$ TRUE $)$. The pressure deviation of $\mathcal{M}_{1}$ comparing

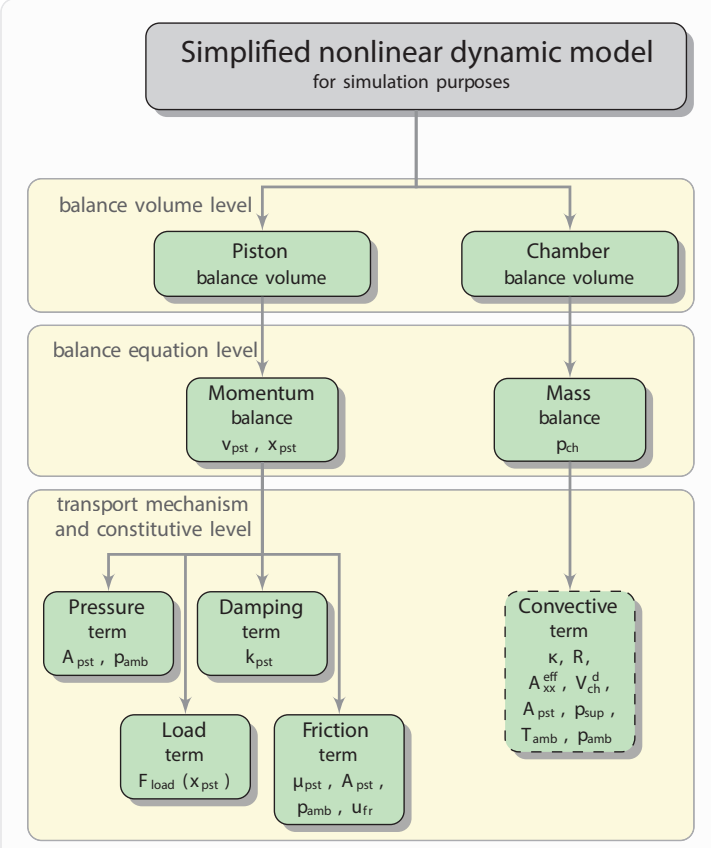

Fig. 6 Hierarchical structure of the simplified nonlinear dynamic model for simulation purposes

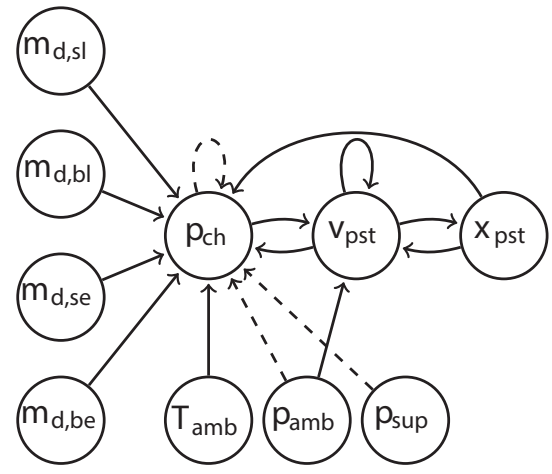

Fig. 7 Structure graph of the differential variables of the simplified model for simulation purposes

with $\mathcal{M}_{0}$ has been increased from $1.23 \%$ to $2.38 \%$, the position deviation has been increased from $2.63 \%$ to $3.43 \%$ and the total deviation has been increased from $2.9 \%$ to $4.18 \%$ hence, the required accuracy has been fulfilled $\left(\chi_{\mathcal{M}_{1} \mathcal{P}_{2}}<\chi_{\mathcal{M}_{1} \mathcal{P}_{2}}^{\max }\right)$.

The number of the balance volumes for which the balance equations are applied has been reduced from 10 to 2 . The hierarchy structure of the simplified model is shown in Fig. 10 where, the term which has hybrid behavior is depicted by dashed line.

The number of the state variables has been reduced from 16 to 3 , the control inputs have been invariant under this simplification process and the disturbance variables has been cut to 3 from the original 5 . The variable structure graph of the simplified model is shown in Fig. 7.

The number of the parameters has been reduced from 83 to 12 where the $F_{l}\left(x_{p s t}\right)$ term, i.e. the center characteristic line of the clutch mechanism, has been considered as a third order polynomial (Hong et al., 2010). The equations have been 


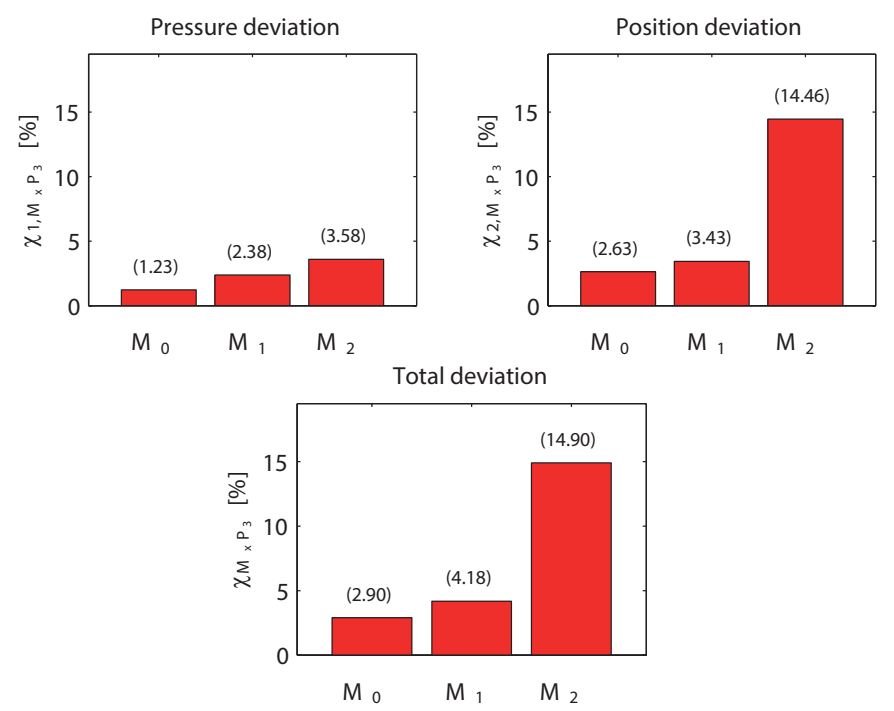

Fig. 8 Model performance indices

simplified considerably by eliminating the power stage / valve subsystem and the gas energy equations hence; the total number of the hybrid terms has been reduced from 12 to 1 . Finally the relative computational time, which corresponds to $1.4 \mathrm{~s}$ time stretch, has been decreased from 35.8 to 12.6. For computing the model size and complexity index the following weights are
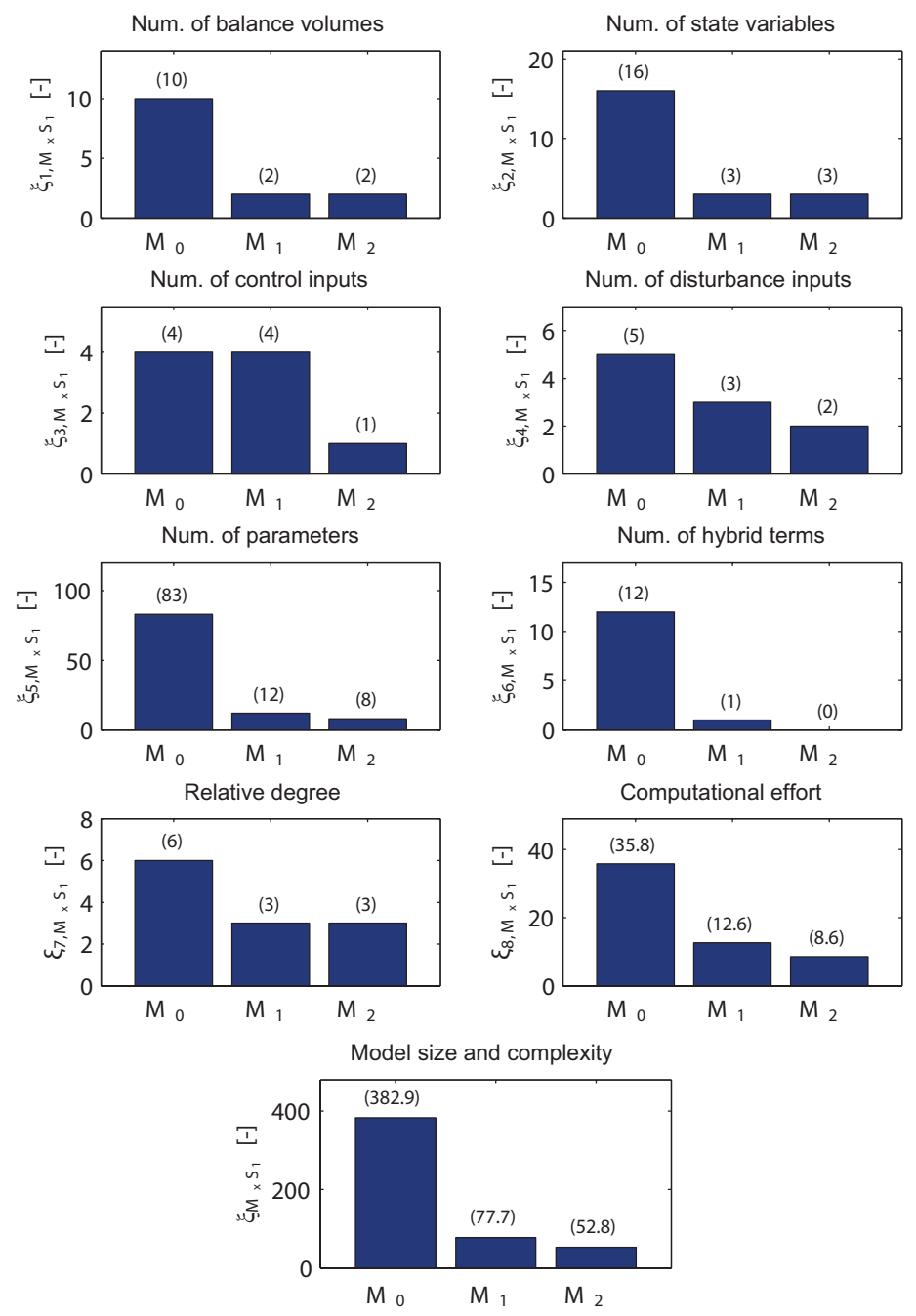

Fig. 9 Model size indices are gathered.

used $w_{1}=4, w_{2}=2, w_{3}=2, w_{4}=1, w_{5}=1, w_{6}=2, w_{7}=1$ and $w_{8}=1$. Through these the model size and complexity index has been decreased from 382.9 to 77.7 . Thus, the simplifying goals have been achieved and the simplifying process has been finished. In Fig. 8 and Fig. 9 the performance and size indices

\subsection{Simplified nonlinear dynamic model for control design purposes $\left(\mathcal{M}_{2}\right)$}

A model for control design purposes should retain all major dynamic characteristics of the real plant (such as its stability and main time constants) but omit all details that are weakly represented in the state variables and not related to the control aims. Hence, the simplified EPC model for control design purposes $\left(\mathcal{M}_{2}\right)$ should has the following properties:

$\mathcal{M}_{2} \mathcal{P}_{1}$ The model variables and parameters should preserve the physical meaning $\left(\chi_{\mathcal{M}_{2} \mathcal{P}_{1}} \in \mathbb{B}\right)$.

$\mathcal{M}_{2} \mathcal{P}_{2}$ The model should be capable of describing the dynamic behavior of the EPC system within $15 \%$ deviation in the whole operation domain $\left(\chi_{\mathcal{M}_{2} \mathcal{P}_{2}} \in \mathbb{R}, \chi_{\mathcal{M}_{2} \mathcal{P}_{2}}^{\max }=0.15\right)$. 
$\mathcal{M}_{2} \mathcal{P}_{3}$ The model should contains only continuous parts i.e. the discrete elements should be omitted or a nominal hybrid mode should be selected $\left(\chi_{\mathcal{M}_{2} \mathcal{P}_{3}} \in \mathbb{B}\right)$.

$\mathcal{M}_{2} \mathcal{S}_{1}$ The model size should be further reduced using the size norm as before $\left(\chi_{\mathcal{M}_{2} \mathcal{S}_{1}} \in \mathbb{R}\right)$.

To achieve these goals above the following assumptions are considered:

$\mathcal{M}_{2} \mathcal{A}_{1}$ Remove the flow property term and use the total mass flow rate of the valves as input.

According to $\mathcal{M}_{2} \mathcal{A}_{1}$ the total mass flow rate of the valves is considered as follows:

$$
\begin{aligned}
\sigma_{v} & =\left(m_{d, s l} A_{s l}^{e f f}+m_{d, b l} A_{b l}^{e f f}\right) \xi_{l} \\
& -\left(m_{d, s e} A_{s e}^{e f f}+m_{d, b e} A_{b e}^{e f f}\right) \xi_{e}
\end{aligned}
$$

This assumption can be done since the dynamic of the flow rate change has similar dynamic as the armature of the valves. This eliminates the hybrid behavior of the model and reduces the number of the inputs from 4 to 1 .

$\mathcal{M}_{2} \mathcal{A}_{2}$ The friction term of the piston can be left out.

This assumption can be done since the friction force is much smaller than the force generated by the pressure.

Applying these assumptions above the state space description of the simplified model for nonlinear control design purposes is obtained. The state vector $\left(\mathbf{x}_{\mathcal{M}_{2}}\right)$ is not changed, the resulted control input vector contains the total mass flow rate of the valves only $\left(\mathbf{u}_{\mathcal{M}_{2}}=\sigma_{v}\right)$ and the disturbance input vector is written as: $\mathbf{d}_{\mathcal{M}_{2}}=\left[p_{a m b} T_{a m b}\right]^{T}$.

The state space description of the model for control design purposes can be written into input-affine model form as follows:

$$
\begin{aligned}
\frac{d \mathbf{x}_{\mathcal{M}_{2}}}{d t} & =\mathbf{f}_{\mathcal{M}_{2}}\left(\mathbf{x}_{\mathcal{M}_{2}}, \mathbf{d}_{\mathcal{M}_{2}}\right) \\
& +\sum_{i=1}^{m} \mathbf{g}_{i, \mathcal{M}_{2}}\left(\mathbf{x}_{\mathcal{M}_{2}}, \mathbf{d}_{\mathcal{M}_{2}}\right) u_{i}
\end{aligned}
$$

where $m$ is the number of the control inputs and the coordinate functions is written as follows:

$$
\begin{gathered}
f_{1, \mathcal{M}_{2}}=-\frac{p_{c h} v_{p s t} A_{p s t}}{V_{c h}^{d}+x_{p s t} A_{p s t}} \\
f_{2, \mathcal{M}_{2}}=\frac{\left(p_{c h}-p_{a m b}\right) A_{p s t}-v_{p s t} k_{p s t}-F_{l}\left(x_{p s t}\right)}{m_{p s t}}
\end{gathered}
$$

$$
\begin{gathered}
f_{3, \mathcal{M}_{2}}=v_{p s t} \\
g_{1,1, \mathcal{M}_{2}}=\frac{\mathrm{R} T_{a m b}}{V_{c h}^{d}+x_{p s t} A_{p s t}} \\
g_{2,1, \mathcal{M}_{2}}=0 \\
g_{3,1, \mathcal{M}_{2}}=0
\end{gathered}
$$

The measured- and the performance outputs are written as the following state-affine equation respectively:

$$
\begin{gathered}
\mathbf{y}_{\mathcal{M}_{2}}=\left[\begin{array}{lll}
1 & 0 & 0 \\
0 & 0 & 1
\end{array}\right] \mathbf{x}_{\mathcal{M}_{2}}=\left[p_{c h}, x_{p s t}\right]^{T} \\
h_{\mathcal{M}_{2}}=\left[\begin{array}{lll}
0 & 0 & 1
\end{array}\right] \mathbf{x}_{\mathcal{M}_{2}}=x_{p s t}
\end{gathered}
$$

The simulation result of this simplified model $\left(\mathcal{M}_{2}\right)$ is shown in Fig. 5 as well. It can be seen that the outputs of $\mathcal{M}_{2}$ have deviance, which mainly caused by the neglected friction.

The most important results of this simplifying procedure are as follows. All the retained model variables and parameters have preserved their physical meaning $\left(\chi_{\mathcal{M}_{2} \mathcal{P}_{1}}=\right.$ TRUE $)$. Since all the discrete switching terms have been eliminated the model became continuous $\left(\chi_{\mathcal{M}_{2} \mathcal{P}_{3}}=\right.$ TRUE $)$. The pressure deviation of $\mathcal{M}_{2}$ comparing with $\mathcal{M}_{1}$ has been increased from $2.38 \%$ to $3.58 \%$, the position deviation has been increased from $3.43 \%$ to $14.46 \%$ and the total deviation has been increased from $4.18 \%$ to $14.9 \%$ (see Fig. 8 . nevertheless, the required accuracy has been fulfilled $\left(\chi_{\mathcal{M}_{2} \mathcal{P}_{2}}<\chi_{\mathcal{M}_{2} \mathcal{P}_{2}}^{\max }\right)$.

The number of the balance volumes has been invariant under this simplification process (see Fig. 9). The hierarchy structure of $\mathcal{M}_{2}$ is shown in Fig. 10 .

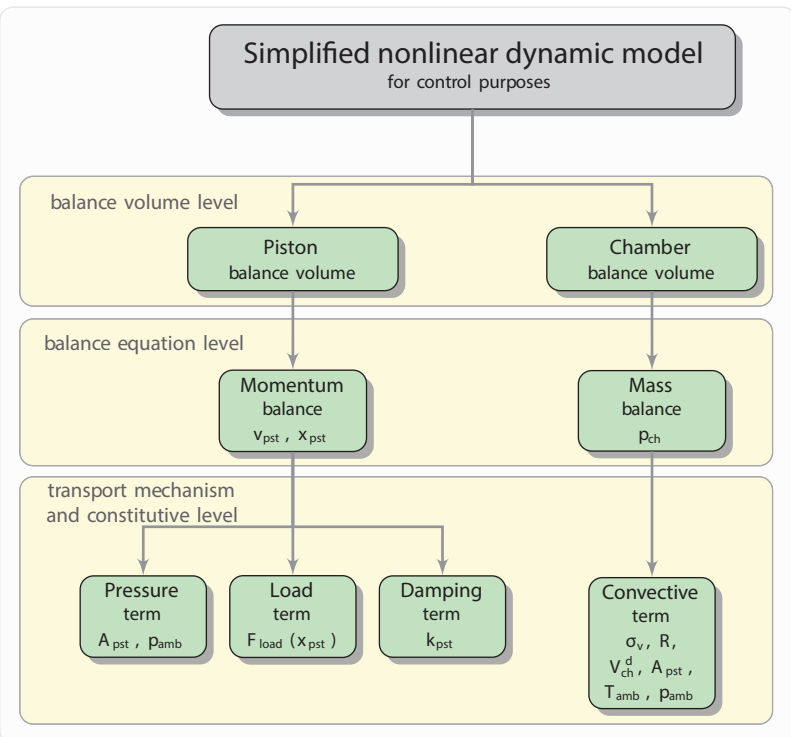

Fig. 10 Hierarchical structure of the simplified nonlinear dynamic model for control purposes 
The number of the state variables has been invariant as well while the control inputs have been reduced from 4 to 1 , the disturbance variables have been reduced from 3 to 2 and the number of the parameters has been reduced from 12 to 8 . The variable structure graph of $\mathcal{M}_{2}$ is shown in Fig. 11. Finally the relative computational time, which corresponds to $1.4 \mathrm{~s}$ time stretch as well has been decreased further from 12.6 to 8.6. Through these the $\mathcal{M}_{2}$ size and complexity index, using the same weights as before, has been decreased from 77.7 to 52.8 . Thus, the simplifying goals have been achieved and the simplifying process has been finished.

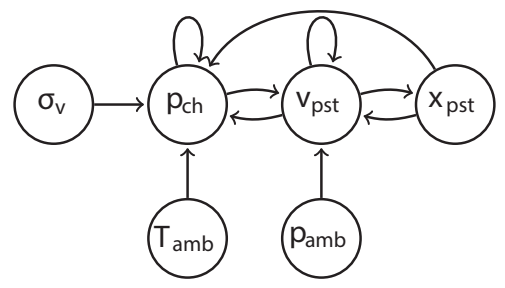

Fig. 11 Structure graph of the differential variables of the simplified model for control purposes

\section{Conclusions}

A systematic approach is applied in this paper for model simplification using engineering judgment and physical insight to find model elements to be left out or simplified. The model simplification is performed by applying simplifying assumptions to the model, which are done in a systematic way using the hierarchy tree of the model. Moreover, performance and size indices are used to decide whether the simplified models fulfill the predefined simplifying goals or not.

The approach is applied for simplifying a dynamic hybrid model of an elecro-pneumatic clutch system that was originally developed from first engineering principles. Using this approach two simplified models are derived. One of them for fast dynamic simulation with reduced computational effort to make it possible to integrate the model into a complete driveline or a vehicle model. The other for control design purposes in which all major dynamic characteristics of the real system are retained and all details that are weakly represented in the state variables and not related to the control aims are omitted.

As one might expect, the complexity of the models are decreased significantly e.g., the number of the states reduced from 16 to 3 . The output errors of the resulted models are increased with the model simplification, nevertheless they remained in the acceptable range. Hence the simplification aims are satisfied with the resulted simplified models. Besides, the model class is changed from discrete-continuous to pure continuous in case of the control oriented model. Hence, the state equations of this model can be written into the standard input affine form which is suitable for control design purposes.

\section{References}

Branicky, M. S., Borkar, V. S., Mitter, S. K. (1998) A unified framework for hybrid control: model and optimal control theory. IEEE Transactions on Automatic Control. 43 (1). pp. 31-45.

DOI: $10.1109 / 9.654885$

Förster, B., Lindner, J., Steinel, K., Strmer, W. (2004) Clutch systems for heavy commercial vehicles. ATZ worldwide. 106 (10). pp. 5-8. DOI: $10.1007 / \mathrm{BF} 03224686$

Förster, B., Steinel, K. (2007) Clutch actuation system ConAct for commercial vehicles with automated manual transmissions. ATZ worldwide. 109 (2). pp. 18-21. DOI: $10.1007 / \mathrm{BF} 03224910$

Hahn, J., Edgar, T. F. (2002) An improved method for nonlinear model reduction using balancing of empirical gramians. Computers \& Chemical Engineering. 26 (10). pp. 1379-1397. DOI: 10.1016/S0098-1354(02)00120-5

Hahn, J., Edgar, T. F., Marquardt, W. (2003) Controllability and observability covariance matrices for the analysis and order reduction of stable nonlinear systems. Journal of Process Control. 13 (2). pp. 115-127. DOI: 10.1016/S0959-1524(02)00024-0

Hangos, K., Cameron, I. (2001) Process modelling and model analysis. San Diego: Academic Press.

Hong, Y., Park, H.-W., Lee, J.-W., Hong, D.-P. (2010) Simulation of clutch actuation system for commercial vehicle. International Journal of Precision Engineering and Manufacturing. 11 (6). pp. 839-843. DOI: $10.1007 / \mathrm{s} 12541-010-0101-5$

Lakner, R., Cameron, I. T., Hangos, K. M. (1999) An assumption-driven case-specific model editor. Computers \& Chemical Engineering. 23. pp. S695-S698. DOI: 10.1016/S0098-1354(99)80170-7

Lakner, R., Hangos, K. M., Cameron, I. T. (2005) On minimal models of process systems. Chemical Engineering Science. 60 (4). pp. 1127-1142. DOI: 10.1016/j.ces.2004.09.074

Laub, A. J., Heath, M. T., Paige, C., Ward, R. (1987) Computation of system balancing transformations and other applications of simultaneous diagonalization algorithms. IEEE Transactions on Automatic Control. 32. pp. 115-122. DOI: $10.1109 /$ TAC.1987.1104549

Leitold, A., Hangos, K. M., Tuza, Zs. (2002) Structure simplification of dynamic process models. Journal of Process Control. 12 (1). pp. 69-83. DOI: 10.1016/S0959-1524(00)00062-7

Moore, B. (1981) Principal component analysis in linear systems: Controllability, observability, and model reduction. IEEE Transactions on Automatic Control. 26. pp. 17-32. DOI: $10.1109 /$ TAC.1981.1102568

Németh, H., Palkovics, L., Hangos, K. M. (2005) Unified model simplification procedure applied to a single protection valve. Control Engineering Practice. 13 (3). pp. 315-326.

DOI: 10.1016/j.conengprac.2004.03.013

Szimandl, B., Németh, H. (2013) Dynamic hybrid model of an electro-pneumatic clutch system. Mechatronics. 23 (1). pp. 21-36. DOI: 10.1016/j.mechatronics.2012.10.006 